\title{
A new control strategy for PMSG based wind turbine to improve power smoothing
}

\author{
Mehrdad Gholami \\ Department of Electrical Engineering. \\ University of Kurdistan \\ Sanandaj, Iran \\ mehrdad.pr@gmail.com
}

\author{
Om-Kolsoom Shahryari \\ Department of Computer Engineering, Sanandaj Branch, \\ Islamic Azad University \\ Sanandaj, Iran \\ shahryari.k@iausdj.ac.ir
}

\begin{abstract}
This paper presents a new simple control strategy for direct driven PMSG wind turbines, using no wind speed sensor. There are several strategies for wind turbine control. Operation of different strategies in terms of power smoothing is compared. New strategy is proposed to have more power smoothing. Performance of the proposed strategy is evaluated by MATLAB/ Simulink simulations and its validity and effectiveness are verified.
\end{abstract}

Keywords: Permanent magnet synchronous generator (PMSG), Power Smoothing, Wind power fluctuation, Wind turbine generator (WTG).

\section{INTRODUCTION}

In recent years, there has been a growing interest in wind energy power systems because of the environmental advantages and the economic benefits of fuel savings [1].Because of intermittency in wind speed, the output power of wind turbine has large fluctuation which may cause frequency deviation in a power grid specially when there are wind farms injecting large amount of power into the grid[2][3]. Therefore, the wind energy conversion systems (WECSs) are increasingly expected to be able to control their output real power, while fully using their capacity, such as retaining their maximum power-point tracking (MPPT) operation [4]. The effect of wind farm penetration into the grid on frequency deviation has been analyzed in [5]. It has shown that very low frequency fluctuation can be compensated by automatic generation control system in conventional power plants. Also the power plant inertias can compensate the high frequency components. The medium frequency components, however, that is to be compensated by governor control system, cannot be compensated effectively. As will be shown, the output power of wind turbine has the low and medium frequency components, but the high frequency component in the wind speed is damped by the turbine inertia. Because of some grid requirements, the output power has to be as smooth as possible. Some methods have been suggested to smooth the output power in the variable-speed wind turbine systems. In one category, the pitch angle control has been employed for smoothening the power fluctuations. To control the pitch angle, the proportional-integral (PI) pitch controller has been often used for the power smoothening [6]. Also some fuzzy logic controller [7-8], predictive controller
[9] have been used to guarantee the power smoothing reliably. These methods, of course, are suitable for wind speeds above the rated value and because of the slow response of the mechanical pitch actuator system; the smoothing cannot be fully accomplished. Also, the need for rapid changes in pitch angle causes mechanical stress on the actuator. Another pitch control method has been proposed for full speed range operation in [10]. While the pitch angle control is necessary above the rated speed to limit the input power, below the rated speed, operation of pitch angle leads to lose maximum power extraction. Also, the slow response of pitch angle mechanical system makes is suitable for low frequency compensation.

Using the DC link voltage control has been suggested for medium frequency compensation in [11]. This method needs extra chopper circuit and causes electrical stress on the DC link capacitor. Since the grid connected inverter requires constant and controllable DC link voltage, so the suggested method might cause some interference in the control objectives.

There are also other methods to control PMSG based wind turbines, such as constant power and constant torque strategies [12]. The constant power strategy needs the value of wind speed to determine the amount of reference power. Also below the rated speed, since the oscillating component of the wind energy is exchanged with the rotor inertia, it may cause large fluctuations in the rotor speed and, in turn, intensive mechanical stresses on the shaft. Furthermore, to have a stable operation in this condition, some reduced value should be selected as the power reference, which results in losing some of the wind power. The constant torque strategy has less rotor speed fluctuations but unlike the constant power method, the power has some fluctuations, in fact the mechanical input power fluctuation is shared between the output and the rotor inertia. Again, similar to the previous methods, it needs the amount of wind speed to determine the reference torque for below the rated wind speed.

Another control strategy that doesn't consider power smoothing issue is the so called MPPT strategy. MPPT strategy is based on maximum power extraction and is done either with wind speed sensor or sensor less. The with sensor method uses the anemometer data and power curves to calculate optimum rotor speed. The sensor less method uses optimum torque or optimum power relations and perturb \& observe algorithm to achieve 
MPPT. Some examples are, DTC control method [1315], DPC control method [16-17], vector control in dq frame [18], power limit search (PLS) algorithm [19]. In these methods both output power and rotor speed have fluctuations according to the wind speed profile. The main advantages of this method are stable operation and maximum power extraction assurance.

In this paper new strategy is proposed to have better power smoothing operation.

In section II different strategies are compared in terms of power smoothing. In Section III, new control method is proposed and a comparison between different methods is made. Then in section IV simulations are carried out and the results are discussed. Finally the conclusion is made in section $\mathrm{V}$.

\section{CONTROL STRATEGIES}

This section allow the authors to describe the problematic proposed in this paper as well literature review regarding the discussed subject

In this section some available strategies are analyzed and compared. The selected methods are Constant power, Constant torque, MPPT and Constant rotor speed. The output powers in these methods are as below (in per unit system):

\section{1) Constant power method \\ 2) Constant torque method \\ 3) Constant rotor speed \\ 4) MPPT method}

In which, $P_{g}$ is the output power and $w_{r}$ is the rotor speed. In direct driven PMSG based wind turbines, since there is no gear box and the shafts of turbine and generator are connected directly, one mass mechanical model can be used:

$$
\begin{gathered}
\frac{d w_{r}}{d t}=\frac{1}{J_{e q}}\left(T_{w}-T_{g}\right) \\
J_{e q} w_{r} \frac{d w_{r}}{d t}=P_{w}-P_{g}
\end{gathered}
$$

For simplicity of analysis the damping term is neglected. The turbine input power by wind is a non-linear function of wind speed $\left(V_{w}\right)$, rotor speed $\left(w_{r}\right)$ and pitch angle $(\beta)$ and is given by (3).

$$
P_{w}\left(V_{w}, w_{r}, \beta\right)=0.5 \rho S V_{w}^{3} C_{p}(\lambda, \beta)
$$

In which, $\rho$ is air density, $\mathrm{S}$ is the area swept by turbine blades of radius $\mathrm{R}, \lambda$ is tip speed ratio $\left(\lambda=\frac{R w_{r}}{V_{w}}\right)$ and $C_{p}$ is a nonlinear wind power coefficient. Because of nonlinearity, small signal analysis is employed to analyze (2). In small perturbation form, it can be written as follows:

$$
J_{e q} w_{r} \frac{d \Delta w_{r}}{d t}=\Delta P_{w}-\Delta P_{g}
$$

From [12], the transfer function for output power in terms of wind speed variation for below the rated speed can be written as:

$$
G_{P_{g} / V_{w}}=\frac{\Delta P_{g}(s)}{\Delta V_{w}(s)}=\frac{\left(3-\frac{\lambda_{0} C_{p 0}^{\prime}}{C_{p 0}}\right)\left(\frac{w_{r 0}}{P_{w 0}}\right) \text { slope }}{\tau_{0} s-\frac{\lambda_{0} C_{p 0}^{\prime}}{C_{p 0}}+\left(\frac{w_{r 0}}{P_{w 0}}\right) \text { slope }}
$$

In which slope $=\frac{\partial P_{g}}{\partial w_{r}},=J w_{0} / P_{w 0}$. From (5), it can be deduced that smaller slope leads to less fluctuation in the output power. This is reasonable because the variation of rotor speed is larger in this condition. Therefore, most of the wind fluctuation is damped by the rotor speed variation. The constant power method has zero gain (Slope $=0$ ). This method produces a constant power, so it is the best in terms of power smoothing, provided that the wind speed is known and the reference power is selected below the available value. This means that the maximum power extracting is lost. Similar to former, the constant torque method needs to wind speed to determining the torque reference and also it has been selected below maximum power condition. For the method of constant rotor speed, as there is no rotor speed variation, all of the wind fluctuation is reflected on the output power. This can be deduced from (5) with Slope $=\infty$.

In the following the MPPT Method is analyzed.

As mentioned before, this method is stable for all regions. The MPPT method can be done in two way, the first one uses lookup table for optimum rotor speed as a reference for the control system(Optimum tip speed ratio), while the other uses cubic function $\left(\mathrm{Pg}=w_{r}^{3}\right.$; optimum power method) [20]. The first method is based on the fact that there is an optimum rotor speed for each wind speed at which maximum power is extracted from wind [6]. Even though both methods are based on maximum power extraction, but the output power traces are different. To illustrate this fact, small signal models for the two ways are compared. For the second way It was presented before (cubic curve) as in (5). Here the small signal model for first the way is extracted. For each wind speed $\left(V_{w}\right)$, there is a rotor speed $\left(w_{o p t}\right)$ at which the power extracted from wind is maximized. According to this fact, the following relation can be defined in per unit.

$$
w_{o p t}=V_{w}
$$

Of course because of high perturbation in the wind speed, it is required to use a low pass filter $\left(\frac{1}{T s+1}\right)$, so (6) is changed as below:

$$
w_{\text {opt }}=\left(\frac{1}{T s+1}\right) V_{w}
$$

According to (4) and (7), the perturbation model for this condition can be written as:

$$
\begin{gathered}
\Delta w_{o p t}=\left(\frac{1}{T s+1}\right) \Delta V_{w} \\
\Delta \mathrm{P}_{\mathrm{w}}=\left(\frac{\partial \mathrm{P}_{\mathrm{w}}}{\partial \mathrm{V}_{\mathrm{w}}}\right)_{\substack{\mathrm{l}_{\mathrm{w}=\mathrm{V}_{0}} \\
\mathrm{w}_{\mathrm{r}=\mathrm{w}_{0}}}} \Delta \mathrm{V}_{\mathrm{w}}+\left(\frac{\partial \mathrm{P}_{\mathrm{w}}}{\partial \mathrm{w}_{\mathrm{r}}}\right)_{\substack{\mathrm{l}_{\mathrm{w}}=\mathrm{V}_{0} \\
\mathrm{w}_{\mathrm{r}}=\mathrm{w}_{0}}} \Delta \mathrm{w}_{\mathrm{r}}
\end{gathered}
$$




$$
\mathrm{G}_{\mathrm{Pg} / \mathrm{V}_{\mathrm{w}}}=\frac{\Delta \mathrm{P}_{\mathrm{g}}}{\Delta \mathrm{V}_{\mathrm{w}}}=\frac{(\mathrm{T} * \mathrm{~A}-\tau) \mathrm{s}+\mathrm{A}+\mathrm{B}}{\mathrm{Ts}+1}
$$

In which; $A=\left(\frac{\partial P_{w}}{\partial V_{w}}\right)_{\substack{V_{w}=V_{0} \\ W_{r}=w_{0}}}, B=\left(\frac{\partial P_{w}}{\partial w_{r}}\right)_{\substack{V_{w}=V_{0} \\ W_{r}=w_{0}}}$.

The bode diagram for gain functions in the two methods are shown in Fig.1. As it is seen, in the first method the wind fluctuation is highly reflected onto the output power, whereas the second method, i.e. the cubic method $\left(P_{g}=w_{r}^{3}\right)$ behaves as a low pass filter and suppresses the high frequency components. Therefore, the second way of MPPT implementation is smoother than the first way. In section $\mathrm{V}$, simulation result of a typical wind speed will be shown to compare these two methods.

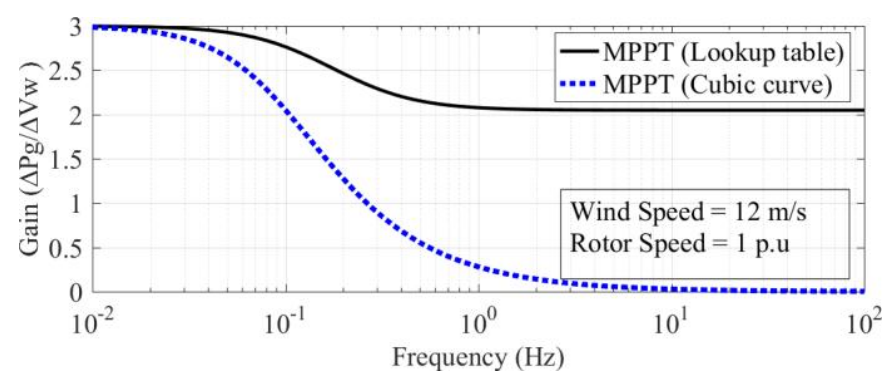

Figure 1 Gain function $\mathbf{G}_{\mathrm{Pg}_{\mathrm{g}} / \mathrm{v}_{\mathbf{w}}}$ in two ways of MPPT method (lookup table and cubic); below rated speed.

\section{PROPOSED METHOD}

This section must include a good technical information to allow the experiments to be repeated. The sources of all media (like name and location of manufacturer) or components of a new formulation must be provided.

According to (5), lesser slope leads to smoother power, therefore, choosing a function with a slope smaller than that of the cubic curve would result in smoother output power. Constant, linear and square functions $\left(P_{g}=\right.$ $a, P_{g}=b w_{r}, P_{g}=c w_{r}^{2}$ ), have lesser slope, but they need the wind speed value to determine their coefficients to have stable operation. On the other hand, functions with higher order such as $P_{g}=w_{r}^{4}$, have more slope than cubic curve, so they cannot be smoother than MPPT method. Therefore, to have stable operation and smoothed power without the need for wind speed value, the only option is using cubic curve with lesser slope. The following function is proposed for this aim.

$$
P_{g}=\alpha w_{r}^{3}, \alpha<1
$$

Since $\alpha<1$, the slope is always less than that of MPPT function. The bode diagram for this method is shown in Fig. 2 for comparison with that of the cubic method.

As it is seen, this method is smoother than MPPT method. In addition, as Fig.3 indicates, this method is always stable because there is an operating point for each wind speed. Lesser value of the coefficient leads to smoother power, but as it can be deduced from Fig.3, there are two problems. First; at rated power the rotor speed exceed the rated value and second; the extracted power is reduced. Therefore, to have smoother power,

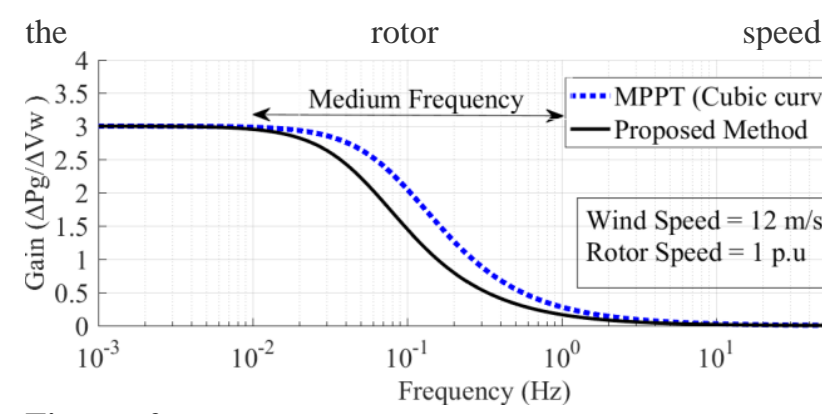

Figure 2 Comparison of gain function $\boldsymbol{G}_{\boldsymbol{P}_{\boldsymbol{g}} / \boldsymbol{V}_{\boldsymbol{w}}}$ for three methods; constant torque, MPPT (Cubic curve) and proposed method; below rated speed region.

should be allowed to exceed the rated value, as well as accepting some reduction in power extraction. In this paper 10 percent rotor over speed is considered to be allowable. According to the power curves, $\alpha=0.7$ is a suitable value for the function coefficient. For this value, as it is observed in Fig. 3, with the increased rotor speed there is insignificant reduction in power extraction as compared to MPPT method.

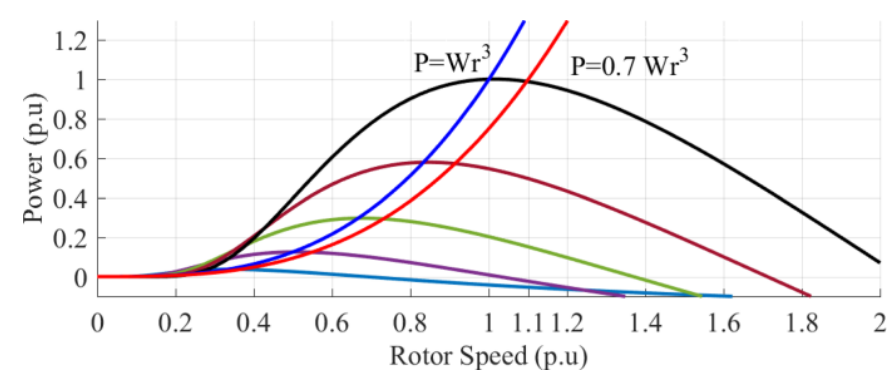

Figure 3 Operating point in MPPT method and proposed method.

Comparison between different methods according to discussions of the previous section is presented in table I. In this table, different methods are compared in terms of power smoothing and stability. Using the proposed method rated speed results in smoother power than that of MPPT method.

\begin{tabular}{|c|c|c|c|}
\hline Method & $\begin{array}{l}\text { Wind } \\
\text { Speed }\end{array}$ & Smoothing & $\begin{array}{c}\text { Power } \\
\text { Capturing }\end{array}$ \\
\hline $\begin{array}{l}\text { MPPT Method (Look } \\
\text { up table) }\end{array}$ & required & weak & MP* \\
\hline $\begin{array}{l}\text { MPPT Method } \\
\text { (Cubic curve) }\end{array}$ & $\begin{array}{l}\text { Not } \\
\text { required }\end{array}$ & Rather good & MP \\
\hline $\begin{array}{l}\text { Proposed Method } \\
\text { (near to cubic) }\end{array}$ & $\begin{array}{l}\text { Not } \\
\text { required }\end{array}$ & good & Near to MP \\
\hline Constant Torque & required & good & $\begin{array}{c}\text { Lower than } \\
\text { MP }\end{array}$ \\
\hline Constant Power & required & Very good & $\begin{array}{l}\text { Lower than } \\
\text { MP }\end{array}$ \\
\hline
\end{tabular}

Table 1: Control methods 


\section{SIMULATION RESULTS}

In this section, include the rationale or design of the experiments as well as the results; reserve interpretation of the results for the Discussion section. Present the results as precisely as possible.

For a 2MW PMSG based wind turbine with the data presented in Appendix [21], simulation is carried out using the proposed control method and conventional method, and the results are compared.

For a typical wind speed profile below the rated value, given in Fig.4, output power obtained by simulation of three methods; MPPT (lookup table and cubic curve) and the proposed method are presented in Fig.5. As it was mentioned in previous section, look up table method has a fluctuated profile. There is a significant difference between the results of lookup table method and the other two methods. It is quite clear that the proposed method results in much smoother output power profile than MPPT methods.

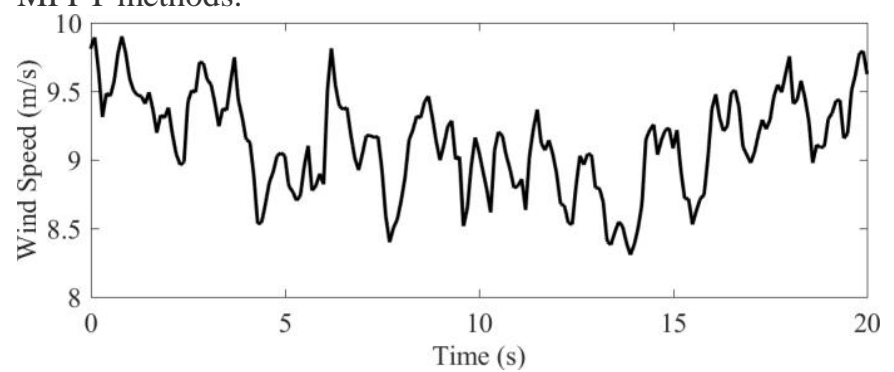

Figure 4 Typical wind speed profile for below rated speed.

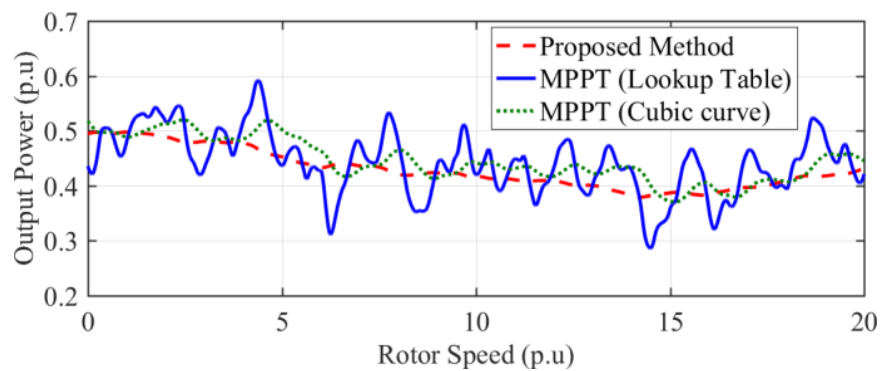

Figure 5 Output power profile for below rated speed in three methods; MPPT (cubic curve and look up table) and proposed method.

\section{CONCLUSION}

In this paper, different control strategies for PMSG based wind turbine at below and above rated speed were compared in terms of power smoothing. Performance of methods were analyzed based on perturbation models. A new control strategy was presented for blow rated wind speed, which presents a smoother output power compared to the MPPT method, while it does not require wind speed information. Furthermore for above rated speed, since the pitch angle controller is slow and cannot set the output power exactly to the rated value, so in this paper another degree of freedom is added to control system. This strategy uses constant torque method for converter control instead of constant rotor speed that is used in conventional strategy. Consequently, the proposed method led to smoother output power and soft operation of pitch angle system.

\section{APPENDIX}

Table 1-A: Wind System Parameters

\begin{tabular}{|l|l|}
\hline \multicolumn{2}{|c|}{ Wind Turbine Parameters } \\
\hline Rated Power & $2 \mathrm{MW}$ \\
\hline Blade Radius & $39 \mathrm{~m}$ \\
\hline Air Density & 1.205 \\
\hline Rated Wind Speed & $12 \mathrm{~m} / \mathrm{s}$ \\
\hline \multicolumn{2}{|c|}{} \\
\hline \multicolumn{2}{|c|}{ PMSG Parameters } \\
\hline Rated Output Power & $2 \mathrm{MW}$ \\
\hline Rated Line-Line Voltage & $690 \mathrm{~V}$ \\
\hline Number of Pole Pairs & 30 \\
\hline Stator Winding Resistance & 0.00344 p.u \\
\hline d-axis Inductance & $0.7685 \mathrm{p} . \mathrm{u}$ \\
\hline q-axis Inductance & $0.4026 \mathrm{p} . \mathrm{u}$ \\
\hline Moment of equal Inertia & $5 \mathrm{p} . \mathrm{u}$ \\
\hline
\end{tabular}

\section{REFERENCE}

[1] Z. Chen, F. Blaabjerg, "Wind farm-A power source in future power systems", Renewable and Sustainable Energy Reviews, vol. 13, no. 6-7, pp. 1288-1300 ISSN: 1364-0321, 2009.

[2] V. Akhmatov, "Analysis of dynamic behavior of electric power systems with large amount of wind power," Ph.D. dissertation, Technical University of Denmark, Kgs. Lyngby, Denmark, Apr. 2003.

[3] L. Jin, S. Y. Zhang, P. Sorensen, L. G. Jie and G. W. Zhong, "Method for Assessing Grid Frequency Deviation Due to Wind Power Fluctuation Based on "Time-Frequency Transformation"," IEEE Trans. Sustainable Energy, vol. 3, no. 1, 2012.

[4] W. Qiao and R. G. Harley, "Grid Connection Requirements and Solutions for DFIG Wind Turbines," IEEE Energy 2030 Conf., Atlanta, GA, USA, pp. 1-8, Feb. 2009.

[5] C. Luo and B. T. Ooi, "Frequency deviation of thermal power plants due to wind farms," IEEE. Energy Conversion, vol. 21, no. 3, pp. 708-716, Sep. 2006.

[6] A. Uehara, A. Pratap, T. Goya, T. Senjyo, A. Yona, N. Urasaki, and T. Funabashi, "A coordinated control method to smooth wind power fluctuations of a PMSG-based WECS," IEEE Trans. Energy Conversion, vol. 26, no.2, pp. 550-558, Jun. 2011.

[7] S. M. Muyeen, J. Tamura, and T. Murata, "Stability Augmentation of a Grid-Connected Wind Farm," London, U.K.: Springer-Verlag, Oct. 2008.

[8] T. L. Van, T. H. Nguyen, D. Ch. Lee, "Advanced Pitch Angle Control Based on Fuzzy Logic for 
Variable-Speed Wind Turbine Systems," IEEE Trans. Energy Conversion, Vol. 30, No.2, Jun. 2015.

[9] M. Soliman, O. P. Malik, D. T. Westwick, "Multiple Model Predictive Control for Wind Turbines with Doubly Fed Induction Generators," IEEE Trans. Sustainable Energy, vol. 2, no. 3, 2011.

[10] A. Uehara, A. Pratap, T. Goya, T. Senjyum, A. Yona, N. Urasaki and T. Funabashi, "A Coordinated Control Method to Smooth Wind Power Fluctuations of a PMSG-Based WECS," IEEE Trans. Energy Conversion, vol. 26, no. 2, Jun 2011.

[11] H. Geng, D. Xu, B. Wu, G. Yang, "Active Damping for PMSG-Based WECS with DC-Link Current Estimation," IEEE Trans. Industrial Electronic, vol. 58, no. 4, Apr 2011.

[12] C. Luo, H. Banakar, B. Shen, B. k. Ooi, "Strategies to Smooth Wind Power Fluctuations of Wind Turbine Generator," IEEE Trans. Energy Conversion, vol. 22, no. 2, JUNE 2007.

[13] Y. Zhao, C. Wei, Z. Zhang, W. Qiao, "A Review on Position/Speed Sensorless Control for Permanent-Magnet Synchronous Machine-Based Wind Energy Conversion Systems," IEEE Journal of Emerging and Selected Topics in Power Electronics, vol. 1, no. 4, 2013.

[14] W. Qiao, X. Yang, X. Gong, "Wind Speed and Rotor Position Sensorless Control for Direct-Drive PMG Wind Turbines," IEEE Trans. Industrial Electronic, vol. 48, no. 1, 2012.

[15] Z. Zhang, Y. Zhao, W. Qiao, L. Qu, "A DiscreteTime Direct Torque Control for Direct-Drive PMSG-Based Wind Energy Conversion Systems," IEEE Trans. Industry Applications, vol. 51, no. 4, 2015.

[16] Y. Xia, K. H. Ahmed, B. W. Williams, "A New Maximum Power Point Tracking Technique for Permanent Magnet Synchronous Generator Based Wind Energy Conversion System," IEEE Trans. Power Electronics, vol. 26, no. 12, 2011.

[17] J. Mohammadi, S. Vaez-Zadeh, S. Afsharnia and E. Daryabeigi, "A Combined Vector and Direct Power Control for DFIG-Based Wind Turbines," IEEE Transactions on Sustainable Energy, vol. 5, no. 3, 2014.

[18] S. Li, T. A. Haskew, K. A. Williams and R. P. Swatloski, "Control of DFIG Wind Turbine with Direct-Current Vector Control Configuration," IEEE Transactions on Sustainable Energy, vol. 3, no. 1, JULY 2012.

[19] J. C. Y. Hui, A. Bakhshai, P. K. Jain, “An Energy Management Scheme With Power Limit Capability and an Adaptive Maximum Power Point Tracking for Small Standalone PMSG Wind Energy Systems," IEEE Transactions on Power Electronics, vol. 31, no. 7, 2016.

[20] M. Nasiri, J. Milimonfared, S. H. Fathi, "Modeling, analysis and comparison of TSR and OTC methods for MPPT and power smoothing in permanent magnet synchronous generator-based wind turbines," Energy Conversion and Management, Volume 86, pp. 892-900, 2014.

[21] B. Wu, Y. Lang, N. Zargary, S. Kouro, "Power Conversion and Control of Wind Energy Systems," New Jersey, Willey-IEEE Press, August 2011. 